\title{
Advanced methods in scanning X-ray microscopy
}

\author{
A. Menzel, ${ }^{a, *}$ M. Dierolf, ${ }^{b, \dagger}$ C. M. Kewish, ${ }^{a}$ P. Thibault, ${ }^{a}$ K. Jefimovs,${ }^{c}$ C. David,${ }^{a}$ M. Bech, ${ }^{d}$ \\ T. H. Jensen, ${ }^{d}$ R. Feidenhans'l, ${ }^{d}$ A.-M. Heegaard, ${ }^{e}$ R. Hansen, ${ }^{e}$ T. Berthing, ${ }^{f}$ K. L. Martinez,${ }^{f}$ \\ J. Als-Nielsen, ${ }^{d}$ S. Kapishnikov ${ }^{g}$ L. Leiserowitz,${ }^{g}$ F. Pfeiffer,${ }^{b, \dagger}$ and O. Bunk ${ }^{a}$ \\ ${ }^{a}$ Paul Scherrer Institut, 5232 Villigen PSI, Switzerland; \\ ${ }^{b}$ Department Physik (E17), Technische Universität München, 85748 Garching, Germany; \\ ${ }^{c}$ EMPA, 8600 Dübendorf, Switzerland; \\ ${ }^{d}$ Niels Bohr Institute, ${ }^{e}$ Department of Pharmacology and Pharmacotherapy, and \\ fDepartment of Neuroscience and Pharmacology \& Nano-Science Center, \\ Copenhagen University, 2100 Copenhagen, Denmark; \\ ${ }^{g}$ Weizmann Institute of Science, Rehovot 76100, Israel.
}

\begin{abstract}
New developments in X-ray instrumentation and analysis have facilitated the development and improvement of various scanning X-ray microscopy techniques. In this contribution, we offer an overview of recent scanning hard X-ray microscopy measurements performed at the Swiss Light Source. We discuss scanning transmission $\mathrm{X}$-ray microscopy in its transmission, phase contrast, and dark-field imaging modalities. We demonstrate how small-angle X-ray scattering analysis techniques can be used to yield additional information. If the illumination is coherent, coherent diffraction imaging techniques can be brought to bear. We discuss how, from scanning microscopy measurements, detailed measurements of the X-ray scattering distributions can be used to extract high-resolution images. These microscopy techniques with their respective imaging power can easily be combined to multimodal X-ray microscopy.
\end{abstract}

Keywords: X-ray microscopy, small-angle X-ray scattering (SAXS), scanning transmission X-ray microscopy (STXM), coherent diffractive imaging (CDI)

\section{INTRODUCTION}

The potential of X-rays for imaging applications was realized within weeks after their discovery. Since the first radiographs, ever new X-ray imaging applications have been utilized, resolution increased, and a variety of different imaging contrast mechanisms employed. The ability to resolve structural details far beyond the resolving power of optical microscopes is due to the significantly shorter wavelength of X-rays. ${ }^{\ddagger}$ All the imaging applications discussed in this communication used wavelength between $0.5 \AA$ and $1.5 \AA$, corresponding to photon energies between $6.2 \mathrm{keV}$ and $18 \mathrm{keV}$. However, none of the techniques presented here depends on this particular energy range. The analysis techniques we discuss are equally applicable to both softer and harder X-rays, and also to other scanning transmission microscopy probes, such as electrons.

We report on new developments in various scanning X-ray microscopy techniques, which were facilitated by $(i)$ the availability of highly brilliant X-ray sources at $3^{\text {rd }}$-generation synchrotrons, $(i i)$ new developments in X-ray detector technology, such as fast-readout area detectors with high dynamic range and low noise characteristics, and (iii) analysis techniques capable of taking advantage of tens of thousands of detailed diffraction patterns for a single specimen. The combination of all of these factors makes possible efficient imaging of extended objects with a spatial resolution given by the extent of the X-ray probe or, using coherent diffraction imaging (CDI) techniques, significantly smaller.

\footnotetext{
* Send correspondence to andreas.menzel@psi.ch.

$\dagger$ until March 2009: Paul Scherrer Institut and Ecole Polytechnique Fédérale de Lausanne, 1015 Laussane, Switzerland

${ }^{\ddagger}$ While techniques exist to overcome the Rayleigh criterion, this entails imaging the distributions of fluorescent markers instead of the objects of interest themselves.
}

Scanning Microscopy 2009, edited by Michael T. Postek, Dale E. Newbury, S. Frank Platek, David C. Joy, Proc. of SPIE Vol. 7378, 73780O - ( 2009 SPIE · CCC code: 0277-786X/09/\$18 · doi: 10.1117/12.821823 
All data presented here have been taken at the coherent small-angle X-ray scattering beamline, cSAXS, at the Swiss Light Source (SLS). The beamline was designed to host a wide variety of sample environments for, inter alia, state-of-the-art small-angle X-ray scattering (SAXS) and coherent diffractive imaging experiments in the energy range of $5-18 \mathrm{keV}$. Fast detection is paramount in scanning microscopy, and thus pixelated detectors, such as charge-coupled devices (CCDs), have only been rarely used in every-day operation. All results reported below were obtained with the PILATUS, a fully pixelated fast framing detector, permanently installed at the cSAXS facility. Each PILATUS pixel acts as a single-photon counter and does not suffer any readout noise. ${ }^{1-3}$ These excellent noise characteristics allow rapid measurements. A PILATUS module, comprising 100000 pixels, can be read out at up to $300 \mathrm{~Hz}$.

\section{SCANNING TRANSMISSION X-RAY MICROSCOPY}

Probably the most common form of scanning microscopy with X-rays is scanning transmission X-ray microscopy (STXM). Typically, a fast point detector is used to measure the X-ray intensity past the specimen. Spatial resolution is gained by tightly focusing the illuminating probe. Resolutions well below $50 \mathrm{~nm}$ have been reported. ${ }^{4-8}$ In case of hard X-rays, necessary to penetrate thick samples, the absorption contrast tends to be rather weak and measuring the phase delay imparted by the specimen often offers significantly higher sensitivity. The absolute phase can be determined by interference or propagation-based techniques, but is experimentally quite challenging to quantify. Yet, differential phase contrast (DPC) is measured readily and quantitatively via the angle of refraction. The DPC signal can be integrated in order to obtain complete knowledge of the complex-valued transmission function of the specimen. ${ }^{9-12}$ This was recognized early on, and more advanced detection schemes than mere transmission measurements were put in place, ${ }^{12-22}$ which frequently also allows the integration of all intensity at $q \neq 0$, where $q$ is the momentum transfer, yielding a dark-field image. ${ }^{\S}$ Using a pixelated detector offers significant flexibility in imaging modes, including the aforementioned absorption, phase contrast, and dark-field, which can all be measured in parallel.

Figure 1 demonstrates diffraction-limited resolution when using a Fresnel zone plate (FZP) as a coherently illuminated focusing device. The FZP used in this demonstration was a $1 \mu \mathrm{m}$ thick gold structure with a diameter of $50 \mu \mathrm{m}$ and a finest zone width of $100 \mathrm{~nm} .^{7}$ The object to be imaged was another zone plate, also featuring $100 \mathrm{~nm}$ structures. The transmission image a) shows limited contrast, but the line profile (along $y=0)$ indicates that even the finest zones are distinguishable. Differential phase contrast, Figs. c) and e), drastically increases contrast. While for the transmission measurement the total detected intensity is used, DPC is measured via the intensity distribution's first moment and does not require a separate measurement. Differential phase contrast is a directional imaging contrast, i.e., in this case it features strong contrast across the imaged zones, but limited contrast along the zones. However, the phase differential parallel to the zones clearly emphasizes connecting bridges between zones, manufactured in order to increase stability of the FZP structure. The increased contrast by DPC is particularly important for weakly scattering specimens, such as life sciences samples. ${ }^{12,22, \text { Sub- }}$ $50 \mathrm{~nm}$ resolution has been achieved using FZPs with a tighter focus and will be published in a forthcoming manuscript. $^{8,23}$

\section{SCANNING SMALL-ANGLE X-RAY SCATTERING}

Whereas typical dark-field imaging analyzes the total amount of scattered light, the combination of scanning Xray microscopy with small-angle X-ray scattering allows spatially resolved analysis of both density and oriention of nano-scale structures. In a scanning SAXS experiment, complete scattering distributions are measured and azimuthally integrated. A fully pixelated detector gives virtually arbitrary freedom when choosing the radial range over which it is integrated, i.e., the length scale to which one chooses to be sensitive. Figure 2 presents a bio-imaging application, namely, collagen-sensitive imaging of a mouse femur, ${ }^{\|}$Figs. d)-f). Other length scales are imaged in parallel.

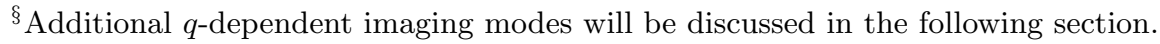

"See also section 4 for STXM imaging of a biological specimen.

${ }^{\|}$Femora were collected from 7 weeks old BALB/c mice. Briefly, the mice were euthanized by cervical dislocation and the femora were removed and post fixed 48 hours in $4 \%$ paraformaldehyde (Lily's solution, Bie\&Bertnsen). Bones
} 

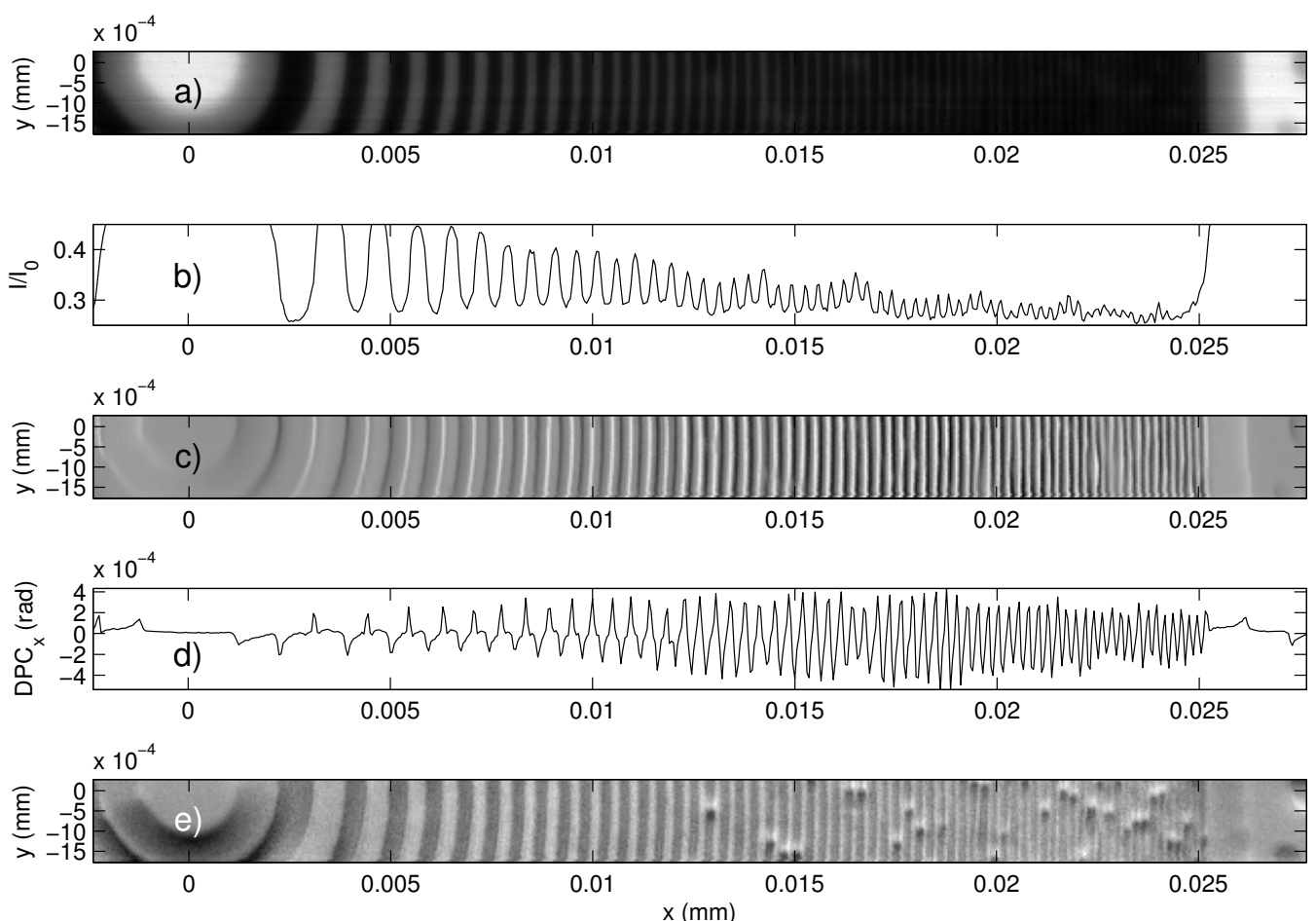

Figure 1. STXM images of a Au Fresnel zone plate. a) and b) transmission contrast, c) and d) DPC in $x$ direction, i.e., horizontal, e) DPC in $y$ direction. The line profiles are taken at $y=0$.

In this specific example, beamline optics focused $10^{11}-10^{12}$ photons per second in a spot of approximately $5 \times 20 \mu \mathrm{m}^{2}$. While the point-to-point resolution does not exceed the resolving power of standard visible-light micrographs, Fig. 3, scattering allows us to gain insight into structures in the nanometer range. Collagen fibres, for instance, scatter anisotropically, and by measuring the orientation of the scattering distribution one can obtain information on the orientation of the fibres. In practice, we integrate over multiple azimuthal segments. Their average intensity can then be Fourier analyzed. ${ }^{24}$ The ratio of first to zeroth Fourier amplitude, i.e., the relative intensity variation as a function of angle, gives a degree of orientation, Figs. 2b), e), and h). The orientation itself holds important information on a variety of life sciences and materials sciences samples. ${ }^{24}$ The outer surface region of the bone exhibits a high degree of orientation on several length scales, attributed to a high degree of ordering in cortical bone.

Standard SAXS analysis yields also information on the dimensionality of the scatterers. Thus, Figs. 2c), f), and i) show spatially resolved fits of the radial profile of the scattering intensity to a polynomial, $I(q) \propto q^{-n}$. The $q^{-1}$ intensity decay predominant in Fig. 2f) is indicative of elongated scattering centers, i.e., collagen fibrils in the present case, and the $q^{-4}$ intensity decay mainly seen in Fig. 2i) shows the presence of more compact, volumetric structures.

Such studies of extended areas in the square millimeter range are routinely performed, and we believe that this technique helps to bridge the gap between high-resolution imaging, which is often restricted to small and thin samples, and large-scale imaging incapable of yielding information on the nanometer scale. SAXS measurements at multiple points have, of course, been performed before. ${ }^{25-30}$ Here, we note the new range of applications offered by the combination of fast read-out detectors and high-brilliance light sources, which allow the acquisition of tens of thousands of diffraction patterns at a rate of several tens of Hertz.

were freed for muscle tissue and decalcified for 3 weeks (15\% EDTA, 0.5\% paraformaldehyde in $0.1 \mathrm{M}$ PBS). Femora were embedded in O.T.C. compound (Tissue-Tek ${ }^{\circledR}$ ) and frozen on a bath of ethanol and dry ice. $100 \mu \mathrm{m}$ sections of the distal femur were cut on a Leica CM $3050 \mathrm{~S}$ cryostat and stored in $0.5 \%$ paraformaldehyde in $0.1 \mathrm{M}$ phosphate buffered saline until measured. 

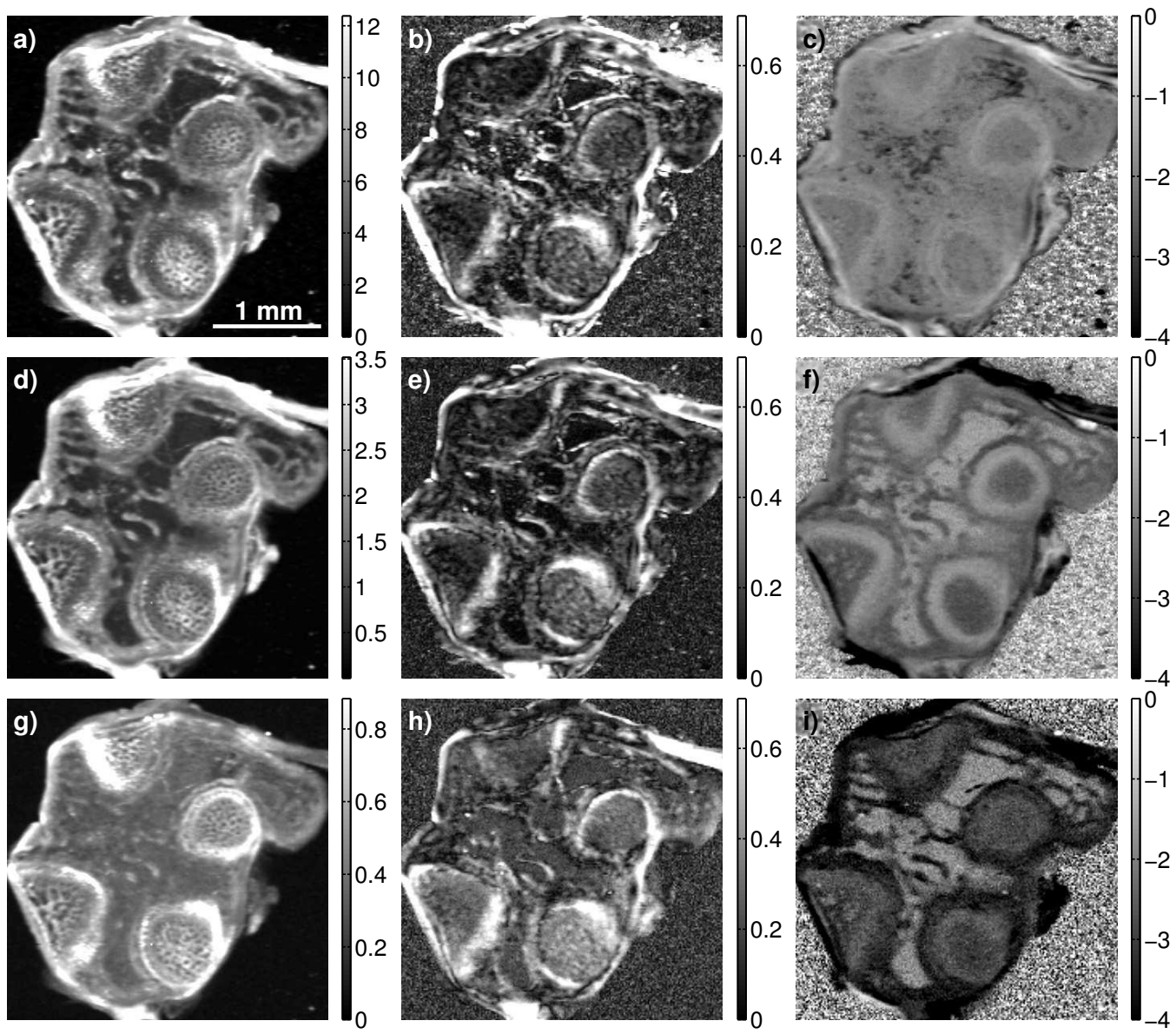

Figure 2. Scanning SAXS images of a mouse fermur section. In each row, three plots are shown for a certain range of length scales. a)-c) correspond to $92.0-143.8 \mathrm{~nm}$, d)-f) to $52.9-76.7 \mathrm{~nm}$, and g) $-\mathrm{i}$ ) to $38.0-47.0 \mathrm{~nm}$. The first column shows the intensity per pixel scattered in this range, the central column shows the degree of orientation, and the right column shows the intensity power law exponent. See text for details.

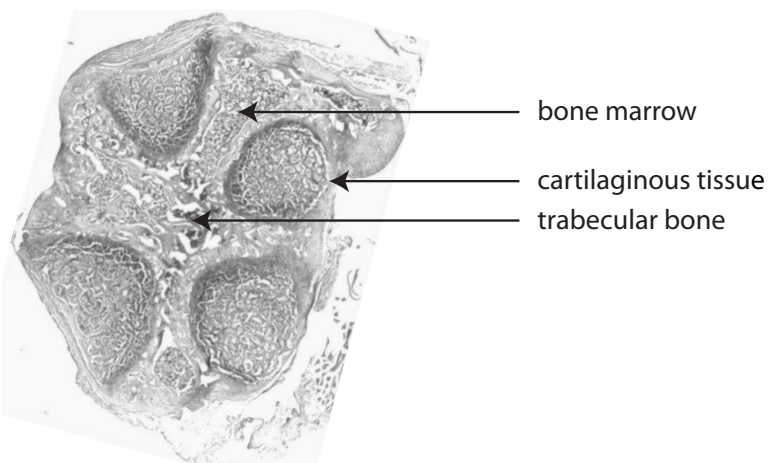

Figure 3. A visible-light micrograph of a stained $6 \mu$ m-thick section. 


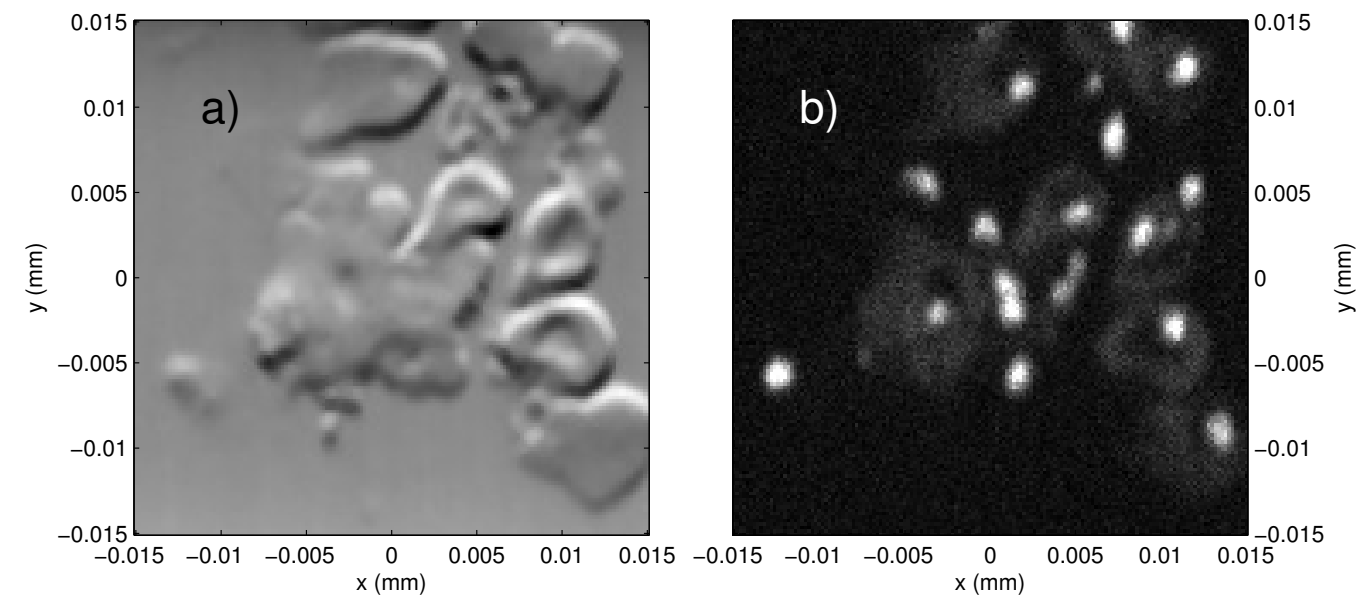

Figure 4. Malaria-infected blood cells. a) STXM image and b) the simultaneously recorded Fe fluorescence. Field of view comprises $30 \times 30 \mu \mathrm{m}^{2}$.

\section{COMPLEMENTARY SCANNING X-RAY IMAGING TECHNIQUES}

Discussions of stability aside, the imaging resolution in scanning microscopy, discussed thus far, is given by the extent of the probe. Furthermore, the location from which imaging information is gained is encoded by motor positions and/or timing. These facts make scanning microscopy inherently adaptable, in that various imaging mechanisms can be exploited in parallel.

As an example, we present STXM and fluorescence imaging results from a malaria-infected blood smear, Fig. 4. The specimen was on a glass "00" cover slide $(\sim 80 \mu \mathrm{m})$, on which were also mounted a labelled transmission electron microscopy (TEM) $\mathrm{Cu}$ grid, in order to easily correlate positions of visible-light micrographs with the X-ray measurements. This was achieved with reduced X-ray flux by prealigning on $\mathrm{Cu}$ fluorescence signals. Photon energy was $9 \mathrm{keV}$, well above the $\mathrm{Fe}$ and $\mathrm{Cu} K$ edges.

The STXM image presented in Fig. 4a) was taken under less than optimal conditions. The sample was not in the focal plane of the focusing FZP, which not only decreased spatial resolution, ${ }^{* *}$ but convolves in a nontrivial way differential phase and absorption contrast. ${ }^{31}$ Nevertheless, unstained biological samples could easily be imaged at photon energies where pure absorption contrast was negligible. Figure 4b) represents integrated counts of the Fe $K \alpha_{1}, K \alpha_{2}$, and $K \beta_{1}$ fluorescence lines over a dwell time of $0.5 \mathrm{~s}$. Both images were taken in parallel, as were scans combining fluorescence mapping and Bragg-reflection-based imaging. The analysis of such scans is ongoing and will be published in a forthcoming article. ${ }^{32}$

\section{SCANNING X-RAY DIFFRACTION MICROSCOPY}

In all the scanning microscopy techniques discussed thus far, the resolution has been limited by the extent of the probe. Manufacturing highly efficient diffractive, refractive, and reflective optics has seen rapid progress, producing imaging optics with ever-increasing resolving power. However, the technological challenges are substantial, and attempts have been undertaken to make independent the imaging resolution from the nano-fabrication techniques required to manufacture the optics. Coherent diffractive imaging techniques have addressed this problem and have seen remarkable success. ${ }^{33-40}$ Such experiments remain challenging, however, relying on inherently chaotic X-ray sources, such as synchrotrons, and requiring stringent conditions on sample mounting or optics characterization.

Scanning X-ray diffraction microscopy (SXDM) is a robust CDI technique, which not only addresses these problems, but has the additional advantage of a sufficiently redundant data set that successful and unique reconstruction of the specimen's complex-valued exit wave is virtually ensured. ${ }^{41,42}$ The technique's origins lay in

\footnotetext{
${ }^{* *} \mathrm{X}$-ray spot size was several hundreds of microns but can be drastically decreased without affecting flux.
} 

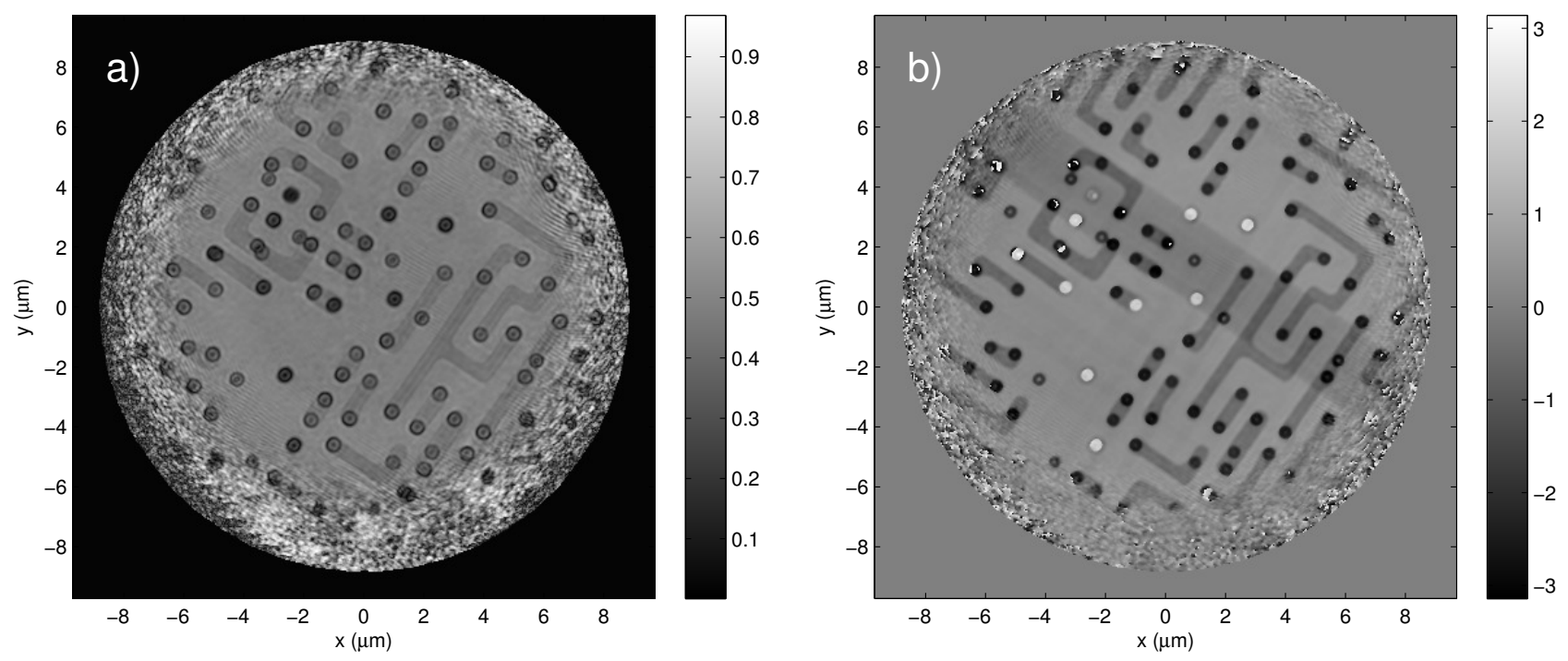

Figure 5. SXDM image of amplitude, a), and phase, b) of a $175 \mu \mathrm{m}$ thick semiconductor chip with integrated circuits (NXP Semiconductors). The phase shows structures, which are not visible in the amplitude.

ptychography, which was developed in the 1960s in the context of electron microscopy. ${ }^{43}$ While the promise of ptychography could be demonstrated, it remained experimentally challenging due to stringent sampling conditions. ${ }^{44-46}$ These were considerably relaxed by merging ptychography with iterative phase retrieval techniques in a technique that was given the name ptychographic iterative engine (PIE). ${ }^{47,48}$ Recently, SXDM was developed to add further robustness to the reconstruction of ptychographic images and make possible additional data extraction beyond imaging the specimen. ${ }^{41,42}$ For instance, SXDM has been used to characterize foci of X-ray optics and promises to exceed in resolution and efficiency most alternative techniques. ${ }^{49,50}$

One of the most salient features of SXDM is its compatibility with standard STXM setups. However, it does not rely on focusing optics, and using a pinhole to define the probe has been found as efficient as using focusing optics in obtaining high-resolution images. ${ }^{51}$ In the studies presented in Fig. 5 , the illumination with a $6.2 \mathrm{keV}$ beam was defined by a tungsten pinhole with $2 \mu \mathrm{m}$ diameter. The figure shows a part of a semiconductor chip and its electrical paths and connections (NXP Semiconductors). Note the significantly enhanced resolution compared to this probe size. Similar enhancement has been achieved in case of a focused X-ray probe. ${ }^{41}$

\section{CONCLUSIONS}

We have illustrated various scanning X-ray microscopy techniques that offer unique opportunities to characterize extended samples on a variery of length scales, ranging from millimeters to a few tens of nanometers. We have demonstrated the compatibility of these various techniques, which is a notable feature of the scanning microscopy approach.

This contribution focused on the imaging aspect of these techniques, while their use in combination with spectroscopic methods can only be mentioned briefly. By choosing photon energies close to the binding energies of electrons, the imaging contrast of X-rays can be made element specific. Even finer spectroscopic control allows the characterization of chemical states of the elements. ${ }^{52}$ By similar means, also magnetic contrast can be gained. ${ }^{53}$ The combination of spectroscopy and microscopy is called spectro-microscopy and has become one of the most salient features of X-ray microscopy. We note that, in case of photon-in-photon-out techniques, i.e., all of the microscopy techniques discussed in this contribution, there is high flexibility in sample environment, which can entail buried structures, extreme temperatures, electromagnetic fields, etc.

Further progress in resolution is expected, both due to improved optics manufacturing and increased efficiency of phase retrieval algorithms. The extensions of all of these techniques to three-dimensional renderings, by standard tomographic methods or by more specialized techniques, are under development. 


\section{ACKNOWLEDGMENTS}

We would like to thank Giovanni Nicolette for his help preparing test samples and Stephanie Stevenson for her assistance during experiments. We further acknowledge gratefully Xavier Donath for his continuous technical support at the cSAXS beamline and thank Philipp Kraft, Eric F. Eikenberry, Beat Henrich, and Christian Brönnimann for PILATUS detector commissioning and support. P.T. acknowledges financial support from the Fonds Québécois de la recherche sur la nature et les technologies (FQRNT).

\section{REFERENCES}

[1] Broennimann, C., Eikenberry, E. F., Henrich, B., Horisberger, R., Huelsen, G., Pohl, E., Schmitt, B., Schulze-Briese, C., Suzuki, M., Tomizaki, T., Toyokawa, H., and Wagner, A., "The PILATUS 1M detector," J. Synchrotron Radiat. 13, 120-130 (2006).

[2] Kraft, P., Bergamaschi, A., Brönnimann, C., Dinapoli, R., Eikenberry, E. F., Graafsma, H., Henrich, B., Johnson, I., Mozzanica, A., Schlepütz, C. M., and Schmitt, B., "Characterization and calibration of PILATUS detectors," IEEE TNS, in print.

[3] Kraft, P., Bergamaschi, A., Broennimann, C., Dinapoli, R., Eikenberry, E. F., Henrich, B., Johnson, I., Mozzanica, A., Schlepütz, C. M., Willmott, P. R., and Schmitt, B., "Performance of single photon counting PILATUS detector modules," J. Synchrotron Radiat., in print.

[4] Chao, W. L., Anderson, E., Denbeaux, G. P., Harteneck, B., Liddle, J. A., Olynick, D. L., Pearson, A. L., Salmassi, F., Song, C. Y., and Attwood, D. T., "Soft 20-nm resolution x-ray microscopy demonstrated by use of multilayer test structures," Opt. Lett. 28(21), 2019-2021 (2003).

[5] Chao, W. L., Harteneck, B. D., Liddle, J. A., Anderson, E. H., and Attwood, D. T., "Soft X-ray microscopy at a spatial resolution better than 15nm," Nature 435(7046), 1210-1213 (2005).

[6] Yin, G. C., Song, Y. F., Tang, M. T., Chen, F. R., Liang, K. S., Duewer, F. W., Feser, M., Yun, W. B., and Shieh, H. P. D., "30 nm resolution x-ray imaging at $8 \mathrm{keV}$ using third order diffraction of a zone plate lens objective in a transmission microscope," Appl. Phys. Lett. 89(22), 221122 (2006).

[7] Jefimovs, K., Bunk, O., Pfeiffer, F., Grolimund, D., van der Veen, J. F., and David, C., "Fabrication of Fresnel zone plates for hard X-rays," Microelectron. Eng. 84(5-8), 1467-1470 (2007).

[8] Jefimovs, K., Vila-Comamala, J., Pilvi, T., Raabe, J., Ritala, M., and David, C., "Zone-doubling technique to produce ultrahigh-resolution x-ray optics," Phys. Rev. Lett. 99(26), 264801 (2007).

[9] Arnison, M. R., Larkin, K. G., Sheppard, C. J. R., Smith, N. I., and Cogswell, C. J., "Linear phase imaging using differential interference contrast microscopy," J. Microsc.-Oxf. 214, 7-12 (2004).

[10] Kottler, C., David, C., Pfeiffer, F., and Bunk, O., "A two-directional approach for grating based differential phase contrast imaging using hard X-rays," Opt. Express 15(3), 1175-1181 (2007).

[11] de Jonge, M. D., Hornberger, B., Holzner, C., Legnini, D., Paterson, D., McNulty, I., Jacobsen, C., and Vogt, S., "Quantitative phase imaging with a scanning transmission X-ray microscope," Phys. Rev. Lett. 100(16), 163902 (2008).

[12] Menzel, A., Kewish, C. M., Dierolf, M., Thibault, P., Bunk, O., Kraft, P., Henrich, B., Eikenberry, E. F., Brönnimann, C., Jefimovs, K., David, C., and Pfeiffer, F., "Scanning transmission X-ray microscopy with a fast framing pixel detector," submitted for publication.

[13] Dekkers, N. H. and de Lang, H., "A detection method for producing phase and amplitude images simultaneously in a scanning transmission electron microscope," Philips Technical Review 37(1), 1-9 (1977).

[14] Hawkes, P. W., "Half-plane apertures in TEM, split detectors in STEM and ptychography," J. Opt.-Nouv. Rev. Opt. 9(4), 235-241 (1978).

[15] Morrison, G. R. and Browne, M. T., "Dark-field imaging with the scanning-transmission X-ray microscope," Rev. Sci. Instrum. 63(1), 611-614 (1992).

[16] Cowley, J. M., "Configured detectors for STEM imaging of thin specimens," Ultramicroscopy 49(1-4), 4-13 (1993).

[17] Morrison, G. R. and Niemann, B., "Differential phase contrast X-ray microscopy," in [X-Ray Microscopy and Spectromicroscopy], Thiema, J., Schmahl, G., Rudolph, D., and Umbach, E., eds., Springer Verlag, Berlin (1998). 
[18] Feser, M., Jacobsen, C., Rehak, P., and De Geronimo, G., "Scanning transmission X-ray microscopy with a segmented detector," J. Phys. IV 104, 529-534 (2003).

[19] Morrison, G., Eaton, W. J., Barrett, R., and Charalambous, P., "STXM imaging with a configured detector," J. Phys. IV 104, 547-550 (2003).

[20] Feser, M., Hornberger, B., Jacobsen, C., De Geronimo, G., Rehak, P., Holl, P., and Struder, L., "Integrating Silicon detector with segmentation for scanning transmission X-ray microscopy," Nucl. Instrum. Methods Phys. Res. Sect. A-Accel. Spectrom. Dect. Assoc. Equip. 565(2), 841-854 (2006).

[21] Kaulich, B., Bacescu, D., Susini, J., David, C., Fabrizio, E. D., Morrison, G. R., Charalambous, P., Thieme, J., Wilhein, T., Kovav, J., Cocco, D., Salome, M., Dhez, O., Weitkamp, T., Cabrini, S., Cojoc, D., Gianoncelli, A., Vogt, U., Pornar, M., Zangrando, M., Zacchigna, M., and Kiskinova, M., "Twinmic - A European twin X-ray microscopy station commissioned at ELETTRA," in [Proc. 8th Int. Conf. X-ray Microscopy], 7, $22-25$ (2006).

[22] Menzel, A., Kewish, C. M., Dierolf, M., Thibault, P., Kraft, P., Bunk, O., Jefimovs, K., David, C., and Pfeiffer, F., "Hard X-ray scanning transmission microscopy with a $2 D$ pixel array detector," J. Phys. Conf. Ser., in print.

[23] Vila-Comamala, J. and David, C., in preparation.

[24] Bunk, O., Bech, M., Jensen, T. H., Feidenhans'l, R., Binderup, T., Menzel, A., and Pfeiffer, F., "Multimodal $\mathrm{X}$-ray scatter imaging,", in preparation.

[25] Paris, O., Žizăk, H., Lichtenegger, I., Roschger, P., Klaushofer, K., and Fratzl, P., "Analysis of the hierarchical structure of biological tissues by scanning x-ray scattering using a micro-beam," Cell. mol. Biol. 46, 993-1004 (2000).

[26] Lorenz-Haas, C., Müller-Buschbaum, P., Wunnicke, O., Cassignol, C., Burghammer, M., Riekel, C., and Stamm, M., "Scanning microfocus small-angle x-ray scattering: A new tool to investigate defects at polymerpolymer interfaces," Langmuir 19, 3056-3061 (2003).

[27] Maier, G., Wallner, G., and Fratzl, P., "3D small angle x-ray scattering (SAXS) on deformed PVDF foils," Mat. Res. Soc. Symp. Proc. 782, 179-185 (2004).

[28] Schroer, C. G., Kuhlmann, M., R. Roth, S. V. G., Stribeck, N., Almendarez-Camarillo, A., and Lengeler, B., "Mapping the local nanostructure inside a specimen by tomographic small-angle x-ray scattering," Appl. Phys. Lett. 88, 164102 (2006).

[29] Stribeck, N., Almendarez-Camarillo, A., Nöchel, U., Schroer, C., Kuhlmann, M., Roth, S. V., Gehrke, R., and Bayer, R. K., "Volume-resolved nanostructure survey of a polymer part by means of SAXS microtomography," Macromol. Chem. Phys. 207, 1139-1149 (2006).

[30] Gourrier, A., Wagermaier, W., Burghammer, M., Lammie, D., Gupta, H. S., Fratzl, P., Riekel, C., Wess, T. J., and Paris, P., "Scanning X-ray imaging with small-angle scattering contrast," J. Appl. Cryst. 40, s78-s82 (2007).

[31] Thibault, P., Dierolf, M., Kewish, C. M., Menzel, A., Viereck, J., and Pfeiffer, F., "Contrast mechanisms in scanning transmission X-ray microscopy,", in preparation.

[32] Dierolf, M., Als-Nielsen, J., Leiserowitz, L., and Pfeiffer, F., in preparation.

[33] Gerchberg, R. W. and Saxton, W. O., "Practical algorithm for determination of phase from image and diffraction plane pictures," Optik 35(2), 237 (1972).

[34] Fienup, J. R., "Reconstruction of an object from modulus of its Fourier-transform," Opt. Lett. 3(1), 27-29 (1978).

[35] Miao, J. W., Charalambous, P., Kirz, J., and Sayre, D., "Extending the methodology of X-ray crystallography to allow imaging of micrometre-sized non-crystalline specimens," Nature 400(6742), 342-344 (1999).

[36] Shapiro, D., Thibault, P., Beetz, T., Elser, V., Howells, M., Jacobsen, C., Kirz, J., Lima, E., Miao, H., Neiman, A. M., and Sayre, D., "Biological imaging by soft x-ray diffraction microscopy," Proc. Natl. Acad. Sci. 102(43), 15343-15346 (2005).

[37] Chapman, H. N., Barty, A., Marchesini, S., Noy, A., Hau-Riege, S. R., Cui, C., Howells, M. R., Rosen, R., He, H., Spence, J. C. H., Weierstall, U., Beetz, T., Jacobsen, C., and Shapiro, D., "High-resolution ab initio three-dimensional x-ray diffraction microscopy," J. Opt. Soc. Am. A 23(5), 1179-1200 (2006). 
[38] Chapman, H. N., Barty, A., Bogan, M. J., Boutet, S., Frank, M., Hau-Riege, S. P., Marchesini, S., Woods, B. W., Bajt, S., Benner, H., London, R. A., Ploenjes, E., Kuhlmann, M., Treusch, R., Duesterer, S., Tschentscher, T., Schneider, J. R., Spiller, E., Moeller, T., Bostedt, C., Hoener, M., Shapiro, D. A., Hodgson, K. O., der Spoel, D. V., Burmeister, F., Bergh, M., Caleman, C., Huldt, G., Seibert, M. M., Maia, F. R. N. C., Lee, R. W., Szoeke, A., Timneanu, N., and Hajdu, J., "Femtosecond diffractive imaging with a soft-x-ray free-electron laser," Nature Physics 2(12), 839-843 (2006).

[39] Pfeifer, M. A., Williams, G. J., Vartanyants, I. A., Harder, R., and Robinson, I. K., "Three-dimensional mapping of a deformation field inside a nanocrystal," Nature 442(7098), 63-66 (2006).

[40] Abbey, B., Nugent, K. A., Williams, G. J., Clark, J. N., Peele, A. G., Pfeifer, M. A., Jonge, M. D., and McNulty, I., "Keyhole coherent diffractive imaging," Nature Physics 4(5), 394-398 (2008).

[41] Thibault, P., Dierolf, M., Menzel, A., Bunk, O., David, C., and Pfeiffer, F., "High-resolution scanning X-ray diffraction microscopy," Science 321, 379-382 (2008).

[42] Thibault, P., Dierolf, M., Menzel, A., Bunk, O., and Pfeiffer, F., "Probe retrieval in ptychographic coherent diffractive imaging," Ultramicroscopy (2009). doi:10.1016/j.ultramic.2008.12.011.

[43] Hegerl, R. and Hoppe, W., "Dynamische Theorie der Kristallstrukturanalyse durch Elektronenbeugung im inhomogenen Primärstrahlwellenfeld," Berichte Bunsen-Gesellsch. 74(11), 1148-1154 (1970).

[44] Rodenburg, J. M. and Bates, R. H. T., "The theory of superresolution electron-microscopy via Wignerdistribution deconvolution," Philos. T. Roy. Soc. A 339(1655), 521-553 (1992).

[45] Nellist, P. D., McCallum, B. C., and Rodenburg, J. M., "Resolution beyond the information limit in transmission electron-microscopy," Nature 374(6523), 630-632 (1995).

[46] Chapman, H. N., "Phase-retrieval X-ray microscopy by Wigner-distribution deconvolution," Ultramicroscopy 66(3-4), 153-172 (1996).

[47] Faulkner, H. M. L. and Rodenburg, J. M., "Movable aperture lensless transmission microscopy: A novel phase retrieval algorithm," Phys. Rev. Lett. 93(2), 023903 (2004).

[48] Rodenburg, J. M., Hurst, A. C., Cullis, A. G., Dobson, B. R., Pfeiffer, F., Bunk, O., David, C., Jefimovs, K., and Johnson, I., "Hard-X-ray lensless imaging of extended objects," Phys. Rev. Lett. 98(3), 034801 (2007).

[49] Guizar-Sicairos, M. and Fienup, J. R., "Measurement of coherent X-ray focused beams by phase retrieval with transverse translation diversity," Opt. Express 17(4), 2670-2685 (2009).

[50] Kewish, C. M., Thibault, P., Dierolf, M., Bunk, O., Menzel, A., and Pfeiffer, F., in preparation.

[51] Menzel, A., Thibault, P., Dierolf, M., Kewish, C. M., Bunk, O., David, C., Leitenberger, W., and Pfeiffer, F., "Advances in ptychographical coherent diffractive imaging," in [Image Reconstruction from Incomplete Data V], Bones, P. J., Fiddy, M. A., and Millane, R. P., eds., Proc. of SPIE 7076, 707609 (2008).

[52] Ade, H., Zhang, X., Cameron, S., Costello, C., Kirz, J., and Williams, S., "Chemical contrast in X-ray microscopy and spatially resolved XANES spectroscopy of organic specimens," Science 258(5084), 972-975 (1992).

[53] Ade, H. and Hsiao, B., "X-ray linear dichroism microscopy," Science 262(5138), 1427-1429 (1993). 